\title{
An Efficient Way to Optimize Bandwidth using Dedicated Path Protection for Sonet/SDH Network
}

\author{
${ }^{1}$ Deepak Dhadwal, ${ }^{2}$ Karishma Bajaj \\ 1, 2(Department of Electronics \& Communication, M.M University, Ambala, Haryana, India) \\ ('deepakdhadwal.007@gmail.com, ${ }^{2}$ vlsi.er86@gmail.com)
}

\begin{abstract}
SONET stands for synchronous optical network which is a time-division multiplexing process (TDMA) for providing bandwidth Services for the end users which works at application level. High Speed data communication and telecommunication traffic are handled with the use of time division technology. For providing efficient high capacity networks required by telecommunication and data services the SONET/SDH standard is used since it uses TDMA for bandwidth services. The Service providers are looking forward to find automatic ways of commissioning the channels associated with the SONET/SDH since manual provisioning is not possible due to high bandwidth requirement. Another thing which is most important is to cover the protection of the optical network in case of failure of any path used for data transfer. An Automatic Path protection algorithm is implemented and moreover analyzed. The algorithm is works for LCAS, VCAT like issues. The Dedicated and shared path protection mechanism has been used for the efficient use of the SONET/SDH network. In this research the basic parameters like probability error, QOS of network, Accepted request and Rejected request have been analyzed by our algorithm. We also investigated the Bandwidth constraint. Using proposed algorithm we have improved the bandwidth utilization.
\end{abstract}

\section{INTRODUCTION}

When information is passed between a sender and receiver, several things must be kept in order on the link, including framing process, error encoding and decoding and the ability of link management. For 1 communications of optical networks these parameters have been standardized by the ANSI T1X1.5 committee which gives the name as Synchronous Optical Networking (SONET) and by the ITU suggest Synchronous Digital Hierarchy (SDH). This paper attempts to tell that how can a SONET/SDH works, down to the application level. For a several years, calls made by telephones are done in the analog method. Calls with longer distance are handled using coaxial cable, twisted pair or analog microwave between various exchanges. In 1960 AT\&T started installation of DS-1 T-carrier services between exchanges with larger distance.. Basically, they are channel banks 2 which has 24 analog circuit and are converted in to digital form and then transmission take place over copper wires to the other exchange centers and are converted back to analog. This method worked extremely well - and it reduces the quantity of copper circuits which is required between exchanges and quality of the telephone calls were improved like less noise and crosstalk. As the distance between sender and receiver increases more, the number of T-carrier circuits required between exchanges increased rapidly. Additionally, Capacity of a circuit can be increased using DS-1C and DS-2 signals. In the late 1980's optical communications became flexible, which allows communications at higher speed which meant that several telephone calls are handled by one circuit connection between sender and receiver. For example, one of the first commercial fiber circuits was installed in France in 1980 and operated at 55 Mbps (DS-3 rate).The terminal multiplexers are installed inside the exchanges. This end to end link is subject to failure - the backup process is provided by the rings which are used in the circuit (Figure 1).In earlier days, the operating companies looked at optical networks as a replacement for the older wire or microwave communications they had been using for years. But then they face a problem of data and power transfer, contactless auto-ID systems are called RFID systems (radio frequency identification) [1]. 


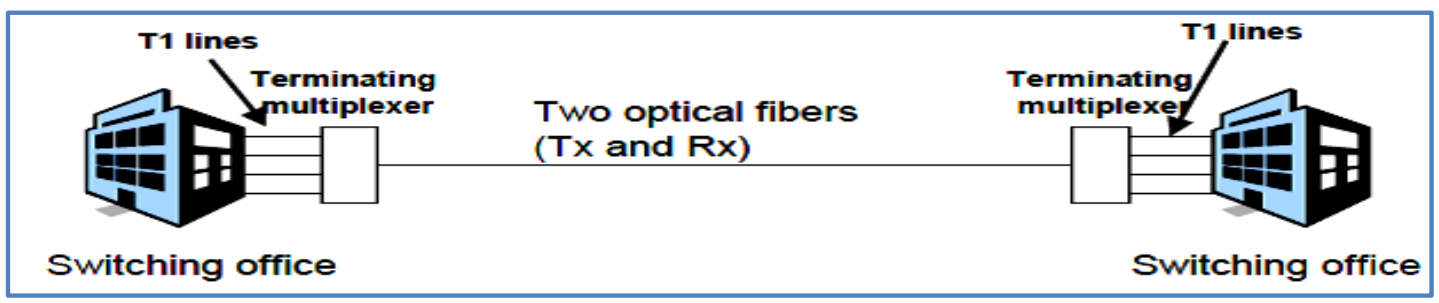

Figure 1: Early use of optical fibers for communication purpose

\section{LITERATURE REVIEW}

As we know that most of the telephone networks infrastructure around the world are based on SONET/SDH technology [1], [2]. SONET adopts a hierarchy of bandwidth structure indicated by STS-n, where $n=1,4,12,18$. STS-I channel is the basic unit in this bandwidth hierarchy, which is equal to $51.84 \mathrm{Mbps}$ of bandwidth. Voice traffic was originally supported by SONET/SDH networks. When the demand for data services began to rise (IP traffic and Ethernet), there is emergency to have cost-effective methods for using various data services in the domain of SONETI/SDH. The role of optical infrastructure is to transfer client traffic between client networks. Circuit traffic can be client traffic, e.g., ATM which stands for asynchronous traffic mode and SDH stands for synchronous digital hierarchy virtual path/virtual channels, or packet traffic, e.g., Internet protocol (IP) packets, which differentiates as traffic flows by recommending equivalent classes. The ILP formulations for the data transfer yield optimal solutions. However, the formulations are very complex and the computational time increases exponentially as the problem size increases. Solving the ILP is not practical for a large sized real world networks. An analysis is made about the current structure of SONET/SDH design issues, the layers, the devices advancements in routing and topology structures. Not only about the Ethernet quality improvement and mapping of the Ethernet frame over SONET frame. The differential delays in a routing path are discussed which is one of the key point for distribution of packet timings. Moreover we can say that we have completely analyzed the current structures of SONET/SDH frame works and layout. With the power of current technology knowledge, now we are able to define the next generation implementation which we have been discussed and optimized. This knowledge of the implementation defines the new algorithms and new devices like Reconfigurable Add Drop Mux, DCSs Routing algorithm at highest optimized level.

\section{PATH PROTECTION MECHANISM AND IMPLEMENTATION}

\section{Dedicated Protection:}

Bandwidth Requirement is one of the critical issue which needs to be solved in the current scenario for high speed communications. This also effects for the network operators and service providers in terms of communication business. That is the reason which forces the Engineers to generated different types of services like DSL, DWDN, SONET/SDH, ATM etc. There is also need of providing several Quality of service for different users. This directly affects the revenue of Service providers.

Dedicated path protection is analyzed, implemented and enhanced for a dense network. A working engine has been created using the Mat lab tool and analysis has been done for multiple requests. The engine is able to hold the network for the static analysis and can provide better and enhanced way for the dedicated protection mechanism. The Engine is also able to handle Multiplexing Hierarchy. The SBPP scheme with the $1+1$ protection scheme in context of CO-OFDM elastic optical networks. For example in fig 3 we can see when a span fail, both techniques the SBPP and $1+1$ protection find a replacing path between the two path end nodes. Assume span (6-8) fails then the working path (1-3-6-8) gets affected and a switch over is performed on to the predefined path (0-2-4-7-8) for a failure recovery. The same happens if span (0-1) fails, working path $(0-1-4)$ gets affected and a switch over is performed onto a predefined route (0-2-4) for recovery failure. Now if we wish to ensure the $100 \%$ failure recovery under $1+1$ protection scheme we need to reserve the same amount of capacity on each protection path as that of its corresponding working path. As shown in figure 25, seven frequency slots (FSs) are reserved on the common span (0-2) and (2-4) which are shared by two protection routes. 


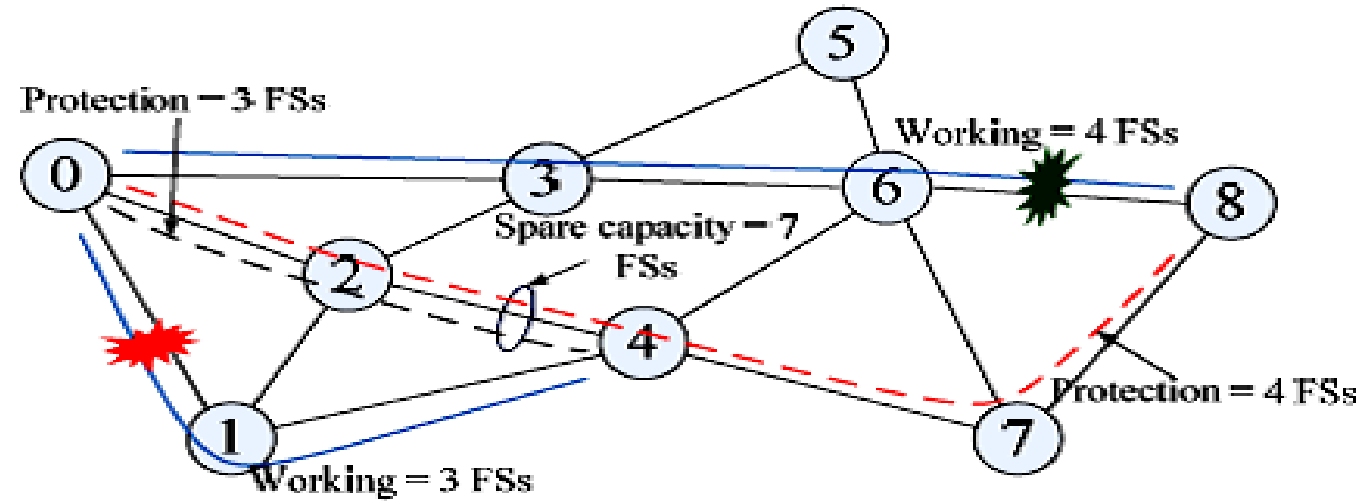

Figure 3: Dedicated Path Protection

There are some points which are required to handle by the network engine for optimization using dedicated method. Process of Simulation consists of two parts:
a. Request Play
b. Network Play

\section{Request Play:}

a. It is used in the frontend.

b. It controls all the generate/process requests

c. Key controls are:

Network Play:
i. Posting a User request
ii. Randomize + Post request (for ease of analysis)
iii. Auto - Request Generator
iv. Randomize request parameters

d. It is a Network Engine which works at the backend

e. It has 2 controls:

i. SONET/SDH State viewer

v. Start/Pause/Resume Network

\section{Request Play:}

f. This is implemented in the frontend.

g. It has all the controls to generate/process requests

h. Key controls are:

vi. Posting a User request

vii. Randomize request parameters

viii. Randomize + Post request (for ease of analysis)

ix. Auto - Request Generator

The Simulations are based on the above points. Network interface provides the interface with the Network which should be specially SONET/SDH network. This network engine runs at back end. It provides the virtual view of the network and we can change the service requests and their bandwidth requirement at our own choice. This help to better analysis on the network. In this engine we can assign number of the nodes and their links with weights automatically or manually. We can stop the running network for the analysis purpose and again resume the network. The states of the network can be check at any time. That how many number of requests handled or rejected by the network at any particular time. This can be check using the state viewer of the Network Engine. 


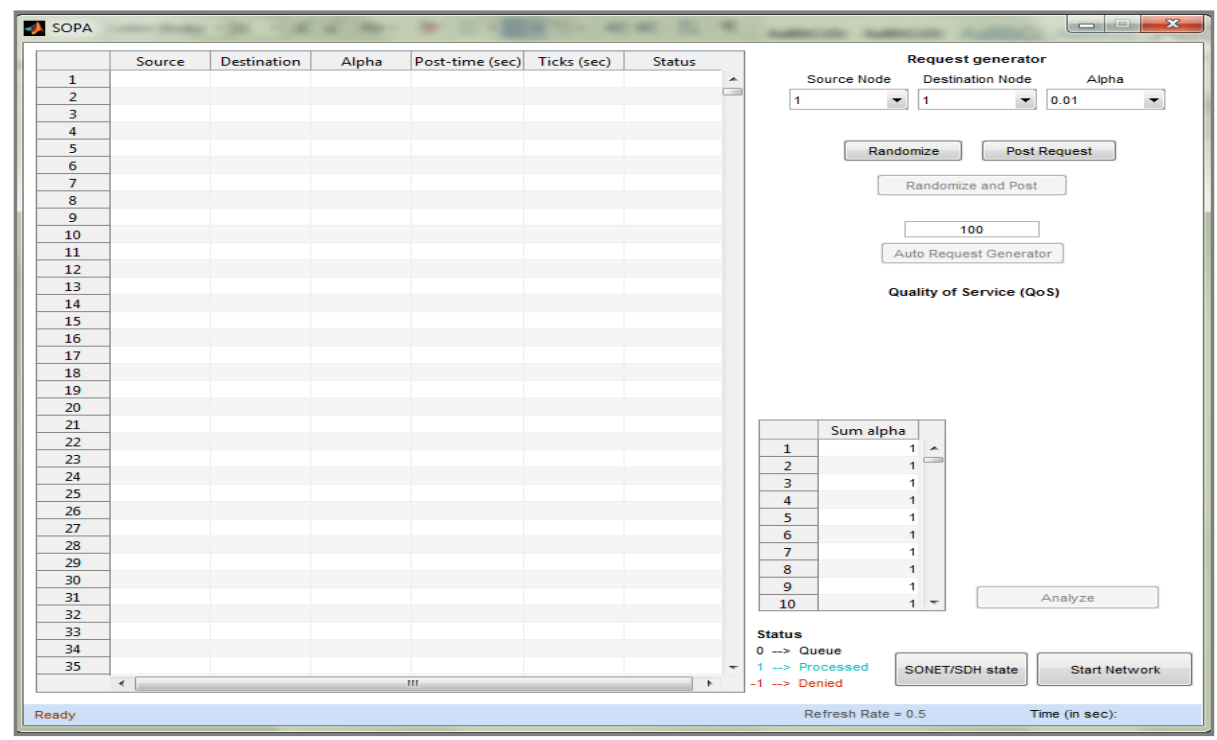

Fig 2: MATLAB Network Engine for Dedicated Path Protection

\section{RESULTS}

Our target is to minimize the no. of rejected/dropped calls in a network this need is to apply the enforcement of engine and bandwidth optimization strategyfor predictive calculations and harness of any unstable situations.

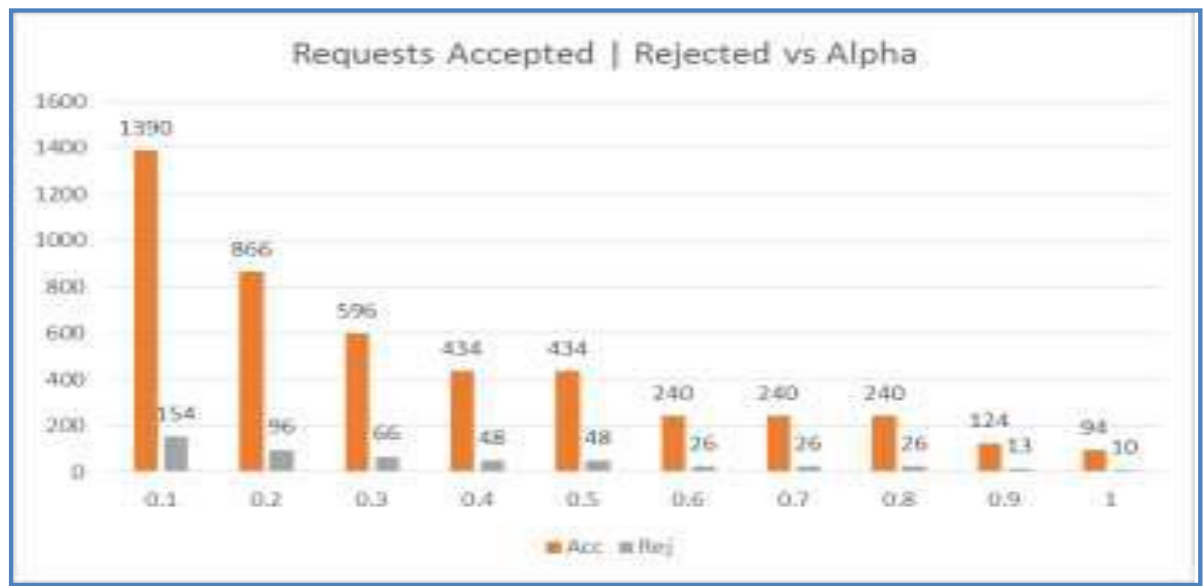

Fig 3: Graph between Requests Accepted /Rejected Vs Alpha 


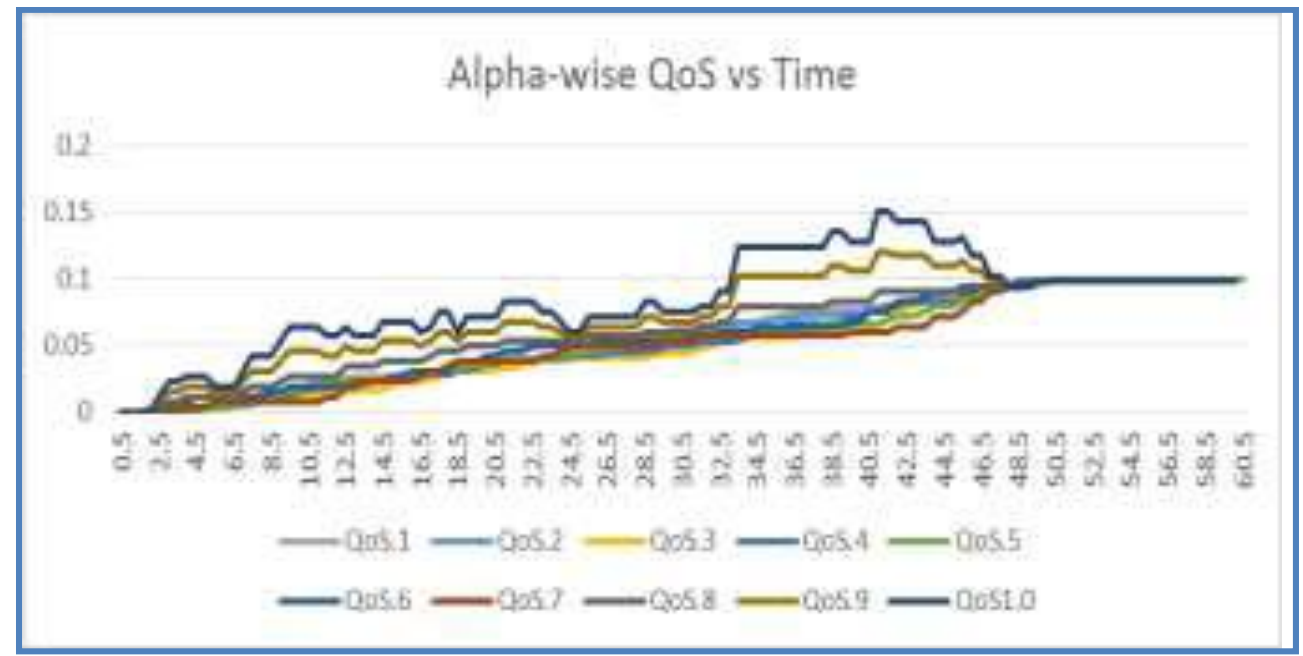

Fig 4: Alpha - Wise Qos Vs Time

- An increase in the call drop rate, the QOS will also increase, and so the network performance reduces.

- First QQS will move with time and then reaches to a constant value.

- QOS will increase for higher value of Alpha, so more Alpha will results to poor performance of the network.

- We take the Alpha value to be very less, then the problem will not be solved as its QoS increases with time in a linear way.

- From the graph, if we select Alpha value to be 0.3 or 0.7, then we got the least effective QoS for the network. On these values system performs at its best.

\section{CONCLUSION}

The necessary features of Advanced SONET that will overcome current limitation are discussed. The key point to realize traffic engineering and to incorporate smart functions such as dynamic bandwidth allocation and high bandwidth utilization are main points of effective path management. The scheme is to provide bandwidth guaranteed path and on disjoint path in order to guarantee QoS as well as to balance network resource utilization. Two practical algorithms are proposed. The resource consumption is minimized by choosing the shortest path and balance the network load by selecting the lightly loaded links. Bellman-Ford algorithm is modified by sequential algorithm to find two disjoint shortest paths one by one. The parallel algorithm finds two disjoint paths by expanding candidate paths to incident links with sufficient bandwidth. Simulation results verify the above algorithms function in normal as well as busy period. Storage space and blocking rate are the two tradeoffs . The study is by far the first one to analyze the dynamic QoS path computation as well as protection for Advanced SONET. At alpha 0.1 the Accepted no. of request is 1102 and Bandwidth utilization is 25. At alpha 0.5 the no. of accepted request enhanced to 1470 out of 1500 which is a very good result. And bandwidth utilization is $32 \%$. At last when alpha is 1 than the No. of accepted request are 1552 out of 1600 and Bandwidth utilization is $33 \%$.

\section{REFERENCES}

[1] N. Ansari, G. Cheng, S. Israel, Y. Luo, J. Ma, and L. Zhu, "QoS provision with path protection for next generation SONET,” in Proc. IEEE ICC, Apr. 2002, pp. 2152-2156.

[2] N. Taft-Plotkin, B. Bellur, and R. Ogier, "Quality-of service routing using maximally disjoint paths,” in Proceedings of IEEE IWQoS '99, 1999, pp. 119-128.

[3] Network node interface for the synchronous digital hierarchy (SDH),.ITU-T Recommendation G.707/Y.1322, Oct. 2000.

[4] Physical/electrical characteristics of hierarchical digital interfaces," ITU-T, Recommendation G.703, 2001.

[5] P. Morreale, K. Sohraby, B. Li, Y. Lin, Guest editorial: “Active, Programmable, and Mobile Code Networking,”'IEEE Communications, Vol. 38, No. 3, pp. 122-123, Mar. 2000.

[6] P.-H. Ho, J. Tapolcai, and H. T. Mouftah, "Diverse routing for shared protection in survivable optical networks," in Proc. IEEE GLOBECOM, 
Dec. 2003, pp. 2519-2523.

[7] Pradhan S., Kusuma J.,and Ramchandran K., "Energy Efficient Data Compression in Wireless Sensor Networks,", Vol. 6, No. 3, pp-297-303, July 2009.

[8] Q. Ma and P. Steenkiste, "On path selection for trafficwith bandwidth guarantees," in Proceedings of 1997 International Conference on Network Protocols, 1997.

[9] Moy, “OSPF version 2,” IETF RFC 2178, July 1997.

[10] Rohith Ramkumar, H.A.Chan, "VCAT Differential Delay Minimization for Delay Sensitive Multiservice Networks", 2006.

[11] R. Madanagopal, N. U. Rani, and T. A. Gonsalves, "Path computation algorithms for dynamic service provisioning in SDH networks," in Proc. 10th IFIP/IEEE IM, May 2007, pp. 206-215

[12] R. Braden, L. Zhang, S. Berson, S. Herzog, and S. Jamin, "Resource ReSerVation Protocol (RSVP)— version 1 functional specification," IETF RFC 2205, Sep. 1997 